\title{
Detection of Hidden Gratings through Multilayer Nanostructures Using Light and Sound
}

\author{
Stephen Edward $\odot,{ }^{1,2,}{ }^{*}$ Hao Zhang $\odot,{ }^{1,3}$ Irwan Setija, ${ }^{4}$ Vanessa Verrina, ${ }^{1,2}$ Alessandro Antoncecchi, ${ }^{1,3}$ \\ Stefan Witte, ${ }^{1,3}$ and Paul Planken ${ }^{1,2, \dagger}$ \\ ${ }^{1}$ Advanced Research Center for Nanolithography, Science Park 106, 1098 XG Amsterdam, Netherlands \\ ${ }^{2}$ Universiteit van Amsterdam, Science Park 904, 1098 XH Amsterdam, Netherlands \\ ${ }^{3}$ Vrije Universiteit Amsterdam, De Boelelaan 1105, 1081 HV Amsterdam, Netherlands \\ ${ }^{4}$ ASML Research, De Run 6501, 5504 DR Veldhoven, Netherlands
}

(Received 20 November 2019; revised 30 March 2020; accepted 18 May 2020; published 7 July 2020)

\begin{abstract}
We report on the detection of diffraction gratings buried below a stack of tens of 18-nm-thick $\mathrm{SiO}_{2}$ and $\mathrm{Si}_{3} \mathrm{~N}_{4}$ layers and an optically opaque metal layer, using laser-induced, extremely high-frequency ultrasound. In our experiments, the shape and amplitude of a buried metal grating are encoded on the spatial phase of the reflected acoustic wave. This grating-shaped acoustic reflection of the buried grating is detected by the diffraction of a delayed probe pulse. A detailed understanding of our measurements is essential for nanometrology applications in the semiconductor manufacturing industry, such as wafer alignment. We show that the complex shape of the diffracted signal as a function of time can be reproduced using a comprehensive numerical model that includes the generation, propagation, and optical detection of the acoustic waves. This allows us to identify the salient features in our measurements such as the presence of acoustic-wave-induced gratings inverted with respect to buried grating, the interference between the optical fields diffracted off multiple grating-shaped acoustic waves, and multiple thin dielectric layers behaving as a single effective acoustic medium. Our results show that laser-induced ultrasound is a promising technique for subsurface nanometrology applications.
\end{abstract}

DOI: 10.1103/PhysRevApplied.14.014015

\section{INTRODUCTION}

In semiconductor device manufacturing, techniques to detect micro and nanostructures buried below the surface of deposited layers are extremely important [1-4]. An example is the detection of so-called alignment gratings. Alignment gratings are gratings etched into Si wafers that are used to position wafers with subnanometer accuracy. This is done by illuminating the grating with a light source and by measuring the diffracted orders emerging from the grating. A small translation of the wafer in the direction of the grating wavevector has no effect on the diffraction efficiency. However, it does change the optical phase difference between the $+n$th and $-n$th diffracted orders (with $n=1,2,3, \ldots)$. A change in the phase difference between, for example, the + first- and -first-order diffracted beams

\footnotetext{
*sedward@arcnl.nl
}

†planken@arcnl.nl

Published by the American Physical Society under the terms of the Creative Commons Attribution 4.0 International license. Further distribution of this work must maintain attribution to the author(s) and the published article's title, journal citation, and DOI. can accurately be detected by interfering the two beams. Measuring these changes makes it possible to align wafers with an accuracy of less than a nanometer [5]. Unfortunately, as device architectures become increasingly complex, alignment gratings can become buried below a large number of dielectric and metallic layers deposited during device fabrication. This poses a huge challenge for the detection of alignment gratings, in particular because some of these layers are completely opaque to light. Fortunately, materials that are opaque to light are often transparent to sound. It has been shown that femtosecond and picosecond laser pulses can be used to generate and detect sound waves with frequencies in the range of tens of gigahertz to several terahertz in solid opaque materials [6-28]. In fact, the ability of acoustic waves to propagate through layers that are not transparent to light, has led to applications in many fields such as biomedical imaging [28-32], material characterization [33-35], defect inspection in manufacturing industry [36], and nondestructive testing [37]. The observation that ultrasound can penetrate materials where light cannot, suggests that laser-induced ultrasound could be used to detect gratings buried underneath optically opaque layers. In one possible implementation of a future photoacoustic alignment sensor, a short laser pulse excites 
an opaque layer above an alignment grating, launching an ultrahigh-frequency acoustic wave. This acoustic wave propagates deeper into the sample and reflects off the buried alignment grating. When the acoustic "copy" of the grating reaches the opaque-layer surface, it can be detected by diffracting light off this grating.

The precision required for alignment applications means that the shape of the diffracted signal as a function of time must be precisely understood. In principle, the individual effects that can contribute to optical diffraction, such as the strain-optic effect, acoustic-wave-induced interface and surface displacement, and possible thermo-optic effect, are well understood. However, the nearly simultaneous occurrence of two or more effects can give rise to diffraction signal shapes and amplitudes that are impossible to predict without an elaborate numerical model. For example, a grating-shaped acoustic wave that flips sign, equivalent to a spatial $\pi$-phase shift, gives rise to a $\pi$-phase shift of the diffracted optical electric field as well. Even if this were the only grating present in the system, knowledge of this sign flip is crucial for wafer alignment, which relies on measuring phase shifts to determine the position of a wafer with sub-nm accuracy [5]. In the presence of different sources of diffraction, interference (constructive or destructive) between the diffracted fields causes changes in the diffraction signal amplitude and can even give rise to subtle changes in the position of diffraction peaks in time. Understanding the origin of the diffracted signal shape from laser-induced ultrasound gratings in complex multilayer systems such as 3D NAND memory [38] is therefore only possible by a comprehensive study that takes all these effects simultaneously into account.

Here, we show how we can detect buried gratings underneath optically opaque layers, by measuring transient optical diffraction from ultrafast, laser-induced, extremely high-frequency acoustic reflections from the grating. In our experiment we make samples consisting of 10-nm amplitude gratings fabricated on top of five or ten bilayers of thin silicon dioxide $\left(\mathrm{SiO}_{2}\right)$ and silicon nitride $\left(\mathrm{Si}_{3} \mathrm{~N}_{4}\right)$ layers on top of a $\mathrm{Au}$ or Ni layer on glass. When viewed from the glass side, the gratings are essentially invisible and can be considered "buried." We perform femtosecond pumpprobe experiments with $400-\mathrm{nm}$ wavelength pump pulses and $800-\mathrm{nm}$ wavelength probe pulses from a Ti:sapphire amplifier, as illustrated in Fig. 1. An optical pump-pulse excites the metal through the glass substrate and launches an acoustic wave, which propagates through the metallic and dielectric layers. The acoustic wave then reflects off the peaks and valleys of the grating and thus acquires a spatially periodic phase. This acoustic copy of the buried grating returns to the glass-metal interface where it deforms the interface in a spatially periodic manner. This grating can be detected by diffraction of a time-delayed probe pulse.

Our measurements show that diffraction is easily observed on these complex multilayer samples. A comparison with calculations shows that a gratinglike deformation at the glass-metal interface induced by the acoustic echo, has an amplitude of several tens of picometers, which is easily detected in our setup. For the sample with ten bilayers of $\mathrm{SiO}_{2}$ and $\mathrm{Si}_{3} \mathrm{~N}_{4}$ layers, after being generated, the acoustic wave has to travel through 42 layers in total before the acoustic echo reaches the glass-metal interface again where it is detected by diffraction of the optical probe pulse. The shape of the measured diffraction signal as a function of time is in good quantitative agreement with the results from numerical calculations performed using the model from Zhang et al. [39]. This model can differentiate between the contributions to the diffraction signal from the displacement of the glass-metal interface and from the strain wave propagating in the glass. These calculations also show why the shape of the diffracted signals versus pump-probe delay and the amplitude of the different diffraction peaks are practically impossible to predict by intuitive means. For example, one would expect that the first grating-shaped acoustic echo to reach the glass$\mathrm{Au}$ interface should give rise to the strongest diffracted signals. For the sample with five bilayers of $\mathrm{SiO}_{2}$ and $\mathrm{Si}_{3} \mathrm{~N}_{4}$ layers, both the experiment and the calculations
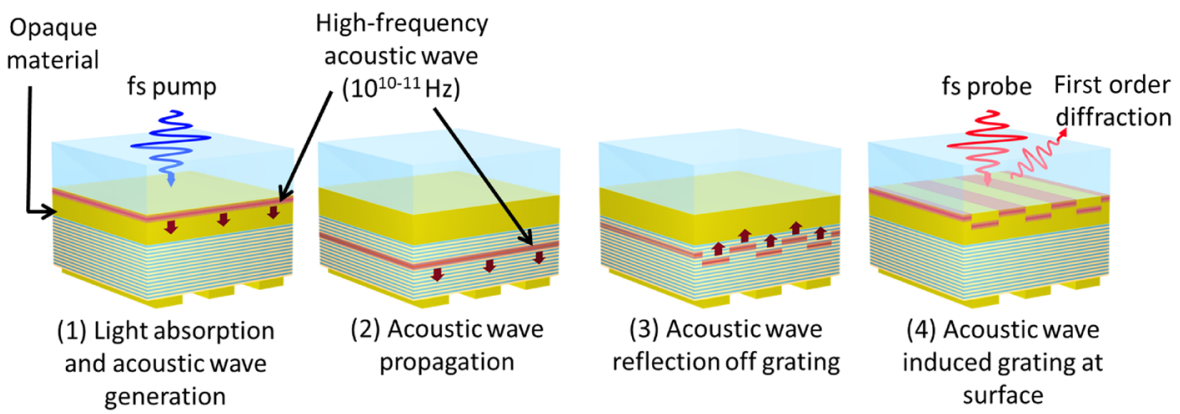

FIG. 1. Schematic explaining the technique. The femtosecond laser pulse is absorbed by the material at the substrate-material interface (1), and launches an acoustic wave that propagates through different layers (2). The acoustic wave reflects off the buried grating and returns to the substrate-material interface as a grating-shaped acoustic wave (3). The time-delayed femtosecond probe pulse diffracts off this interface grating, and the first-order diffraction signal is recorded (4). 
show that the second peak is, in fact, stronger. We also find that the strongest peak in the diffracted signal is the result of diffraction off an acoustic-wave-induced grating that is inverted with respect to the buried grating. This gives rise to a $\pi$ shift of the optical phase of the firstorder diffracted fields compared to a noninverted grating. This is essential information for wafer alignment, which relies on measurements of the optical phase. In addition, such phase shifts lead to destructive or constructive interference with the optical fields diffracted off acousticwave-induced gratings in the glass, which can strongly decrease or increase the amplitude of the diffracted signal. Understanding these effects is crucial for a waferalignment application in an industry environment where a quantitative understanding of the signal is required to obtain a high measurement accuracy. We find that replacing the $\mathrm{SiO}_{2} / \mathrm{Si}_{3} \mathrm{~N}_{4}$ layer stack with a single layer having an equivalent time-averaged sound velocity and average density, has only a small effect on the shape and amplitude of the diffracted signal as a function of time. Our results show that buried gratings can be detected through optically opaque layers on complex, multilayered samples, using laser-induced, extremely high-frequency ultrasound. This technique shows promise as a noncontact, all-optical, subsurface nanostructure detection and imaging modality.

\section{EXPERIMENTAL DETAILS}

A schematic of the pump-probe setup used for the experiments is shown in Fig. 2. A Ti:sapphire multipass amplifier generates $30-\mathrm{fs}$ pulses with a repetition rate of $1 \mathrm{kHz}$ and with a wavelength centered at $800 \mathrm{~nm}$. The output from the laser is split into two by a $95 / 5$ beamsplitter. A $100-\mu \mathrm{m}$ beta barium borate (BBO) crystal frequency doubles the stronger beam to generate 400 -nm pump pulses. The weaker part of the $800-\mathrm{nm}$ beam is used as a probe.

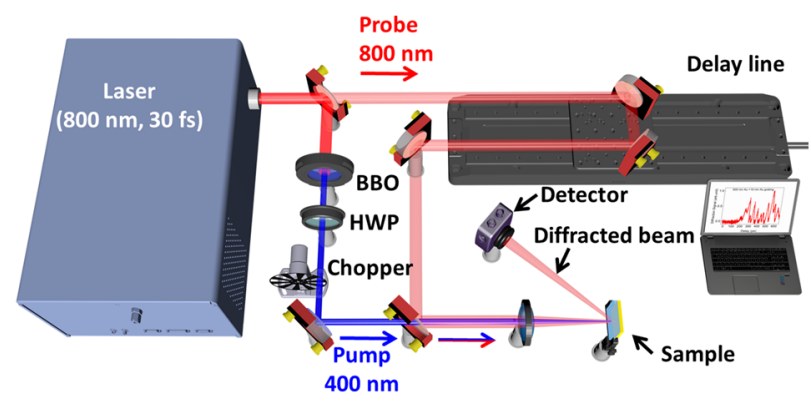

FIG. 2. Schematic of the experimental setup. 30-fs pulses with a wavelength of $800 \mathrm{~nm}$ are split into pump and probe beams. The pump is frequency doubled in a BBO crystal, passes through a half-wave plate (HWP) and is focused onto the sample with a lens. The $800-n m$ probe beam passes through a variable optical delay line and is focused onto the sample on the same spot as the pump beam. An optical detector measures the first-order diffracted probe beam.
A half-wave plate rotates the polarization of the $400-\mathrm{nm}$ pump pulse by $90^{\circ}$ so that both the pump and the probe are $p$ polarized. Both the pump and probe are weakly focused onto the sample. The pump beam diameter is about $500 \mu \mathrm{m}$ and pump pulse energy ranges from 6 to $8 \mu \mathrm{J}$ depending on the sample. The probe beam diameter is $250 \mu \mathrm{m}$ and the probe pulse energy is kept constant at $2 \mu \mathrm{J}$. A silicon photodetector is placed at the position where the first-order diffracted probe beam from the buried grating is expected. The signal recorded by the detector when the chopper blocks the pump beam is subtracted from the diffracted probe signal when the pump beam is transmitted by the chopper, and plotted as a function of the pump-probe delay.

In a recent publication [40], we demonstrated that a similar experimental setup could be used to detect gratings buried underneath a single thin Au layer (approximately $100 \mathrm{~nm}$ ) by optical measurements of ultrafast electron dynamics. In contrast, in the present paper, we discuss laser-induced ultrasound measurements on much more complex samples for which ultrafast electron dynamics could never be used to detect a buried grating. A brief explanation and discussion on the advantages of using laser-induced ultrasonics can be found in Appendix A.

\section{RESULTS AND DISCUSSION}

\section{A. Detection of grating under metal layers}

Prior to experiments on samples with metallic and multiple dielectric layers, we first perform pump-probe experiments on relatively simple samples consisting of (i) a 10 -nm amplitude $50 \%$ duty-cycle Au grating on a $522-\mathrm{nm}$ flat Au layer deposited on glass [Fig. 3(a)] and, (ii) a 10-nm amplitude, $50 \%$ duty-cycle Ni grating on a $315-\mathrm{nm}$ flat $\mathrm{Ni}$ layer deposited on glass [Fig. 3(b)]. Our choice for Au as the grating and layer material is motivated by the fact that $\mathrm{Au}$ is one of the most well-studied materials. However, it has a relatively small electron-phonon coupling constant compared to that of $\mathrm{Ni}$, and this has a significant impact on the shape of the resulting acoustic signals $[41,42]$.

In Fig. 3(c), we plot the measured first-order diffracted signal (red curve) as a function of the pump-probe delay for the sample consisting of a 10-nm amplitude Au grating on a 522-nm flat Au layer on glass. The graph shows that for the first $150 \pm 2$ ps after optical excitation, there is no diffracted signal. After this, a diffraction signal emerges that slowly rises to a maximum at a delay of around $280 \pm 2$ ps. A second maximum can be seen around delay values of about $560 \mathrm{ps}$. Superimposed on these is a more rapidly oscillating signal with a period of $47 \pm 2$ ps. Note that the signature of this more rapid oscillation is already present on the rising edge of the first main diffraction peak but also on the falling edge of the second main diffraction peak. We want to point out that the very observation 


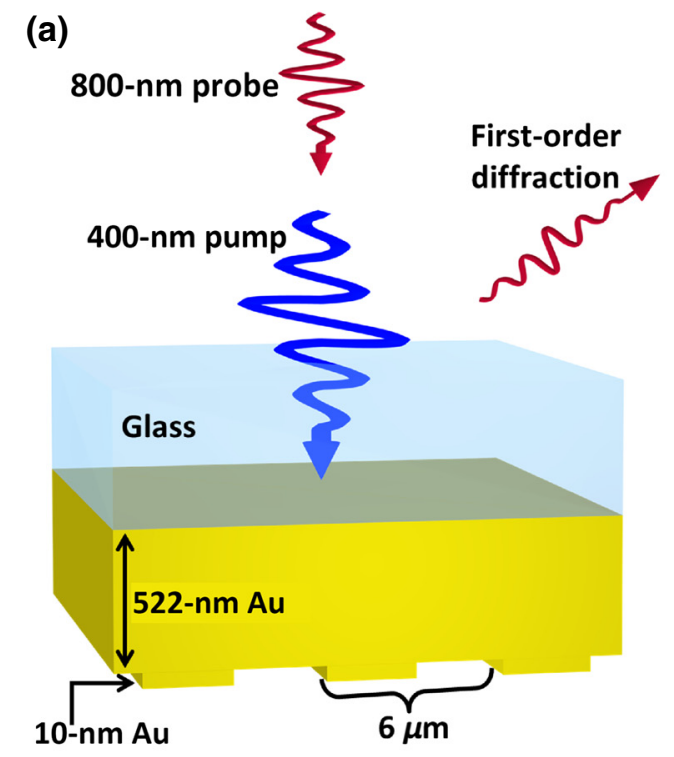

(c)

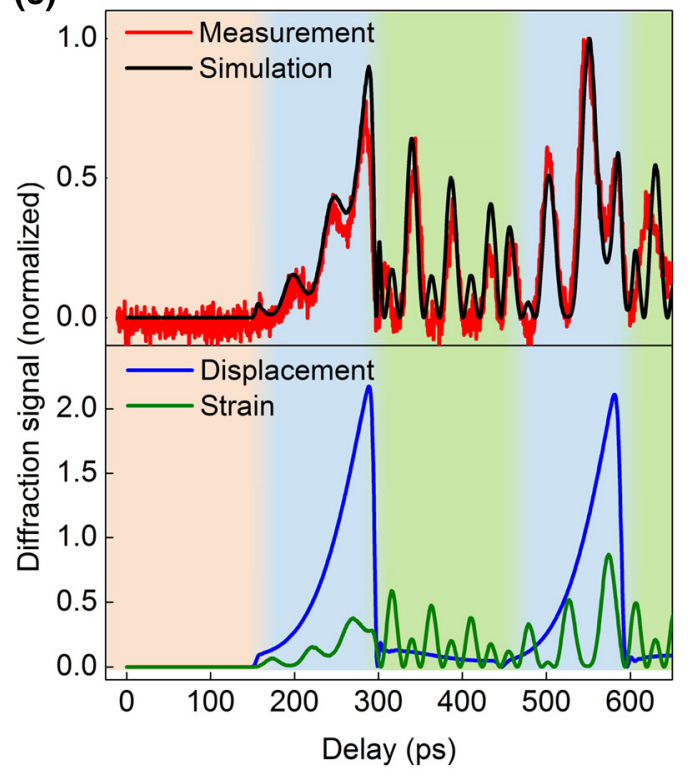

(b)

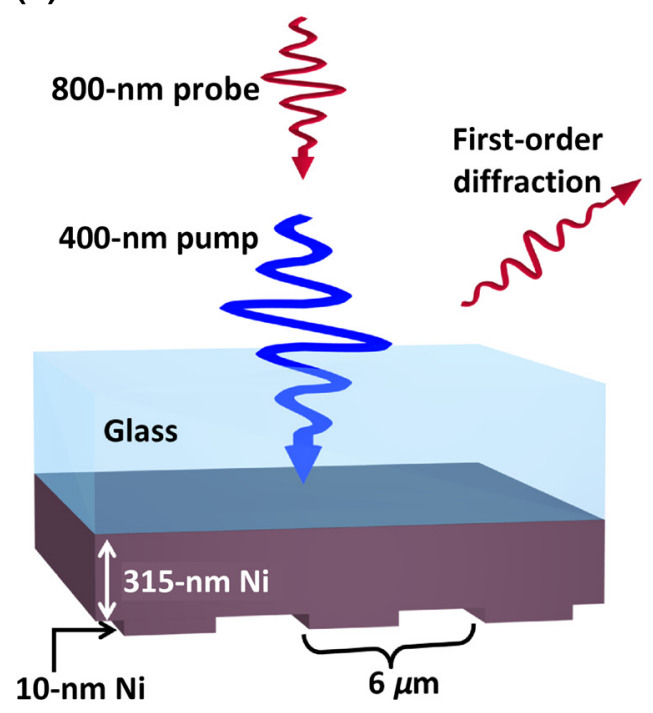

(d)

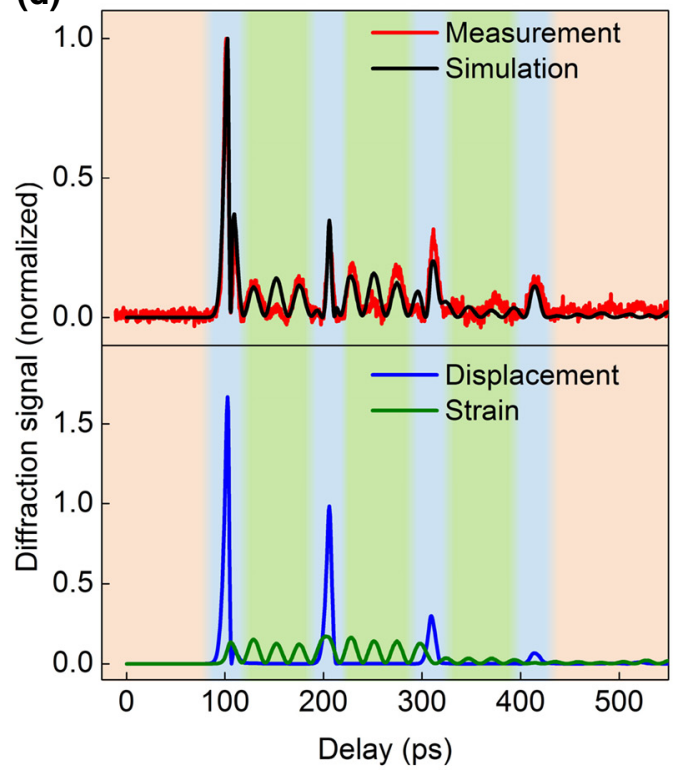

FIG. 3. Schematics of the beam and sample geometry for (a) the 10-nm amplitude Au grating on a 522-nm Au layer on glass and (b) $10-\mathrm{nm}$ amplitude Ni grating on a $315-\mathrm{nm} \mathrm{Ni}$ on glass. Both gratings have a 50\% duty cycle. The pump pulse has a wavelength of 400 $\mathrm{nm}$ and the probe pulse has a wavelength of $800 \mathrm{~nm}$. (c) Upper panel: experimentally measured (red) and numerically simulated (black) diffracted probe signal versus pump-probe delay for the $\mathrm{Au}$ on Au grating sample. Bottom panel: calculated probe-diffraction signal versus pump-probe delay taking only the displacement of the glass-Au interface into account (blue line), or taking only the propagating strain pulse in the glass substrate into account (green line). (d) Upper panel: experimentally measured (red) and numerically simulated (black) diffracted probe signal versus pump-probe delay for the $\mathrm{Ni}$ on $\mathrm{Ni}$ grating sample. Bottom panel: calculated probe diffraction signal versus pump-probe delay taking only the displacement of the glass-Ni interface into account (blue line), and taking only the propagating strain pulse in the glass substrate into account (green line).

of diffraction after 150 ps implies the presence of a grating at the glass-Au interface. This grating is caused by the returning acoustic wave whose wavefront has the topography of the buried grating imprinted on it through a spatially periodic delay in the acoustic arrival time, or phase.

To properly understand these measurements we first focus on the acoustic wave generation mechanism in Au.
The hot electron gas created after optical excitation rapidly diffuses deeper into the thick Au layer to a maximum depth of several hundred nanometers $[40,41,43]$. On a time scale of a few picoseconds, the electron gas cools by heating the lattice. The highest lattice temperatures are found near the glass- $\mathrm{Au}$ interface, where the light is absorbed. The rapid heating of the lattice leads to a rapid expansion of 
the lattice, which launches a longitudinal acoustic pulse with a spatial extent corresponding to the electron energy diffusion depth. The slow, early rise of the diffraction signal versus time is due to the arrival of part of the acoustic wave that has been generated deep inside the Au layer and thus is the earliest to reflect off the buried grating. The diffraction signal continues to slowly increase up to $280 \pm 2$ ps at which time the part of the acoustic wave that is generated near the glass-Au interface returns to the interface again after reflecting off the buried grating. A second, broad diffraction signal is seen around 550-580 ps, which represents the arrival of the same acoustic wave after the second round trip. The two broad signals ( $280 \mathrm{ps}$ and $550-580 \mathrm{ps}$ ) are the result of diffraction from a gratinglike displacement of the atoms at the glass-Au interface due to the returning acoustic echo. For other materials, spatially periodic changes in the optical constants of the materials, caused by changes in material density induced by the sound wave, can also contribute. As we show later, for $\mathrm{Au}$, our calculations indicate that displacement of the glass- $\mathrm{Au}$ interface leading to a grating with an amplitude of up to $110 \mathrm{pm}$ at the glass-Au interface is the dominant cause of diffraction.

The rapid, lower amplitude oscillation superimposed on the signal is surprising, considering that its period does not match the round-trip propagation time in 522-nm Au. This can be explained as follows: the grating-shaped acoustic echo at the glass-Au interface also shows partial transmission into the glass and continues to propagate away from the interface. The wavefront of this acoustic echo is still spatially periodic, modifies the refractive index of the glass, and gives rise to probe-pulse diffraction. Normally, the presence of such a quasistatic grating in glass would give rise to a constant (in time) diffracted signal. Here, however, the same probe pulse that diffracts off this grating also reflects from the glass-Au interface and then diffracts off the grating in the same direction again. The extra optical phase acquired by the propagation of the optical pulse before it diffracts a second time leads to interference between the electric fields of the diffracted beams. Whether the interference is constructive or destructive depends on the distance that the optical pulse has propagated before diffracting again. This in turn depends on the distance that the acoustic echo in the glass has travelled from the glass-Au interface and is thus a periodic function of time [44-48]. This phenomenon is often referred to as Brillouin scattering. The diffraction signal we measure is the coherent sum of the optical fields diffracted off the grating-shaped acoustic wave at the Au-glass interface and off the grating-shaped acoustic wave in the glass.

The Brillouin scattering in glass dominates the timedependent diffraction signal we observe between 300 and $450 \mathrm{ps}$. The diffraction peak at $283 \pm 2 \mathrm{ps}$ is from the first round trip of the acoustic wave, and the peak at $580 \pm 2$ is from the second round trip of the acoustic wave in 522-nm $\mathrm{Au}$, assuming an acoustic velocity of $3200 \mathrm{~m} / \mathrm{s}$ in Au. The diffraction signal we observe after $450 \mathrm{ps}$ therefore is a coherent sum of diffracted signals from multiple contributions, namely (i) light diffracting from the grating-shaped acoustic echo in $\mathrm{Au}$ when it is close to the glass-Au interface after the second round trip, (ii) light diffracting from the grating-shaped acoustic wave that entered the glass after the first round trip and, (iii) light diffracting from the grating-shaped acoustic wave that entered the glass after the second round trip. Interference between the light beams diffracted off these gratings makes it difficult to predict what the temporal shape of the diffracted signal will look like. We therefore resort to two-dimensional (2D) numerical simulations of the generation, propagation, diffraction, and optical detection of the acoustic waves, which take into account all the aforementioned contributions. The simulations are based on a numerical model we develop, which solves a set of equations by the 2D finite-difference timedomain method. Those equations describe the generation, propagation, and optical detection of the acoustic waves. The model includes diffraction caused by the displacement of the surface at the glass-Au interface, diffraction by possible changes in the complex refractive index in the metal, and diffraction of light by the refractive-index grating formed by the acoustic waves in the glass. The model is briefly explained in Appendix B and details of the model can be found elsewhere [39].

In Fig. 3(c) (upper panel), we plot the numerically simulated diffraction signal as a function of the pump-probe delay for the 10-nm amplitude grating on 522-nm Au on glass (black curve). In the bottom panel, we plot the calculated diffracted intensity taking only the displacement of the glass-Au interface by the acoustic wave into account (blue curve), and the diffracted intensity calculated taking only the changes in the optical constants of the glass due to the strain-optic effect, or Brillouin scattering, into account (green curve). Note that the full calculations (black curve) cannot simply be obtained by adding the curves calculated for the strain contribution (green curve), to that for the curve calculated for surface atom displacement only (blue curve). The reason for this is that the phases of the diffracted light scattered by the two types of gratings have to be taken into account as well. This can give rise to destructive interference and thus a net diffracted signal that is smaller than the numerical sum of the intensities of the diffracted beams obtained for each grating separately. The model calculates the diffracted optical field to obtain the diffracted intensities, thus those coherent additions are fully taken into account.

To study the effect that the electron-phonon coupling strength has on the shape of the acoustic signal reflected off the buried grating, we also perform measurement on a 10-nm amplitude Ni grating fabricated on a flat 315nm-thick Ni layer on glass [Fig. 3(b)]. In Fig. 3(d) we plot the measured first-order diffracted signal as a function of the pump-probe delay for this sample (red curve). The 
diffraction signal stays zero for the first $100 \mathrm{ps}$ after optical excitation, after which it rises sharply within 5 ps in contrast to the results shown for $\mathrm{Au}$ where the signal increases more slowly. The diffraction peaks we observe at $102 \pm 2$ ps, $205 \pm 2$ ps, $310 \pm 2 \mathrm{ps}$, and $415 \pm 2$ ps are due to diffraction of the probe pulse after the first, second, third, and fourth round trip of the acoustic echo that originated at the glass-Ni interface, respectively.

In Fig. 3(d) (upper panel), we plot the numerically calculated diffraction signal (black curve) along with the measured diffraction signal for the Ni sample. In Fig. 3(d) (lower panel), we plot the diffraction signals due to (i) the displacement of the atoms near the glass-Ni interface (blue curve) and (ii) Brillouin scattering in the glass substrate (green curve). Here too, the calculated diffraction signal that takes both surface displacement and Brillouin scattering into account (black curve), is very similar to the measured one. These calculations allow us to unambiguously attribute the largest diffraction peaks at 102, 205, 310 , and 415 ps to scattering off the spatially periodic displacement of the atoms at the glass-Ni interface after each round trip of the acoustic wave inside the Ni layer. The lower amplitude diffraction peaks observed in between are caused by Brillouin scattering in the glass.

We observe that the diffraction peaks are "sharper" than for the "Au-multilayer" sample, and this can be explained by the larger electron-phonon coupling constant of $\mathrm{Ni}$, which leads to shorter acoustic waves. The electronphonon coupling constant in $\mathrm{Ni}$ is about 12 times larger than that of Au (see Table I). In Ni, the hot electron gas formed after optical excitation cools much more rapidly and heats the lattice before the hot-electron-gas energy can diffuse much deeper into the layer. The resultant acoustic wave is much more localized in the propagation direction than in the case of $\mathrm{Au}$ and thus gives rise to more narrow peaks in the diffraction signals.

Another noticeable feature in this measurement is the very sharp decrease of the diffraction signal at $102 \mathrm{ps}$, immediately after the first main diffraction peak. The sharp dip can be understood from the shape of the wavefront of the acoustic wave after it reflects off the buried grating. The part of the wave that reflects off the valleys of the grating takes approximately $3.5 \mathrm{ps}$ longer to return to the glass-Ni interface than the part that reflects off the peaks of the grating. In fact, we can view the two parts as two separate time-delayed gratings, shifted by half a grating period, equivalent to a $\pi$-phase shift, in the direction parallel to the grating vector. When both gratings are close to the interface, within the region that corresponds to the optical penetration depth of the probe pulse, the probe can diffract off both gratings simultaneously. However, a $\pi$-phase difference between the two gratings implies a $\pi$-phase difference between the electric fields diffracted off the two gratings. Therefore, soon after the arrival of the first acoustic grating, the second acoustic grating arrives, and the field diffracted off this grating destructively interferes with that diffracted off the first. This leads to a very sharp dip in the diffracted intensity immediately after the first diffraction peak. Similar effects are also observed for the diffracted signal of the Au sample with a buried grating. These features are characteristic for optical diffraction experiments from acoustic waves reflected off real gratings, and the diffraction signals are difficult to quantify without resorting to extensive numerical calculations. Note also that these effects do not occur in transient-grating experiments in which a gratingshaped acoustic echo is created by two temporally and spatially overlapping pump pulses, which form an interference pattern $[9,13,17]$. There, only one grating-shaped acoustic wave is present because in the dark fringes of the interference pattern no acoustic wave is generated.

A second diffraction peak is observed at 109 ps. In the calculations of the signal diffracted by the interface displacement only [blue curve in Fig. 3(d)], this second diffraction peak is barely visible as a small peak immediately following the dip. In the full calculation (black curve), the coherent addition of the fields diffracted by the interface displacement grating and the strain-optic effect in the glass leads to a relatively strong enhancement of the peak. This means that the signal cannot be understood from the simple addition of the calculated intensities of the signal diffracted by the interface displacement grating only and by the Brillouin scattering only. The phases of the grating and thus of the diffracted electric fields have to be taken into account as well. Similar effects can be observed at 206 ps, where the light diffracted from the displacement grating destructively interferes with the light diffracted due to the Brillouin scattering in the glass, resulting in the reduction of the total diffracted intensity.

Between 110 and 190 ps, we observe diffraction peaks caused by Brillouin scattering in the glass substrate. In contrast to the measurement on the Au sample described above, diffraction peaks caused by Brillouin scattering in glass are not observed on the rising edge of the diffracted signal from the first acoustic echo in the Ni layer. This is because the rise time of the acoustic wave amplitude is simply too short. By the time the acoustic wave arrives near the glass-Ni interface and is partially transmitted by it, the acoustic wave in the glass has not propagated far enough to observe the first peak. This is different for the Au case where the acoustic wave is much longer.

After about 115 ps, the acoustic echo is no longer present at the glass-Ni interface, and the small diffraction peaks at 130 and 176 ps are only due to Brillouin scattering in the glass. The separation between these peaks is 46 ps, which is exactly the Brillouin oscillation period in glass for the probe wavelength used. Similarly, after the second round trip of the acoustic wave in Ni at $205 \mathrm{ps}$, we observe small diffraction peaks separated by 46 ps at $230 \pm 2$ and $276 \pm 2$ ps, which are also due to the Brillouin scattering. 
The short duration of the acoustic wave in $\mathrm{Ni}$ enables us to more easily separate the Brillouin scattering effect in the glass from that of the acoustic wave in the metal.

We observe that the width (FWHM) of the diffraction peaks due to the acoustic wave in $\mathrm{Ni}$ at 102, 205, 310 , and $415 \mathrm{ps}$, is $5 \pm 1,7 \pm 1,9 \pm 1$, and $13 \pm 1 \mathrm{ps,}$ respectively. This gradual increase in the FWHM of the diffraction signal after each round trip is suggestive of acoustic dispersion of the acoustic wave. Purely from the measurements, it is difficult to ascertain whether acoustic dispersion is the only factor contributing to the increase in the FWHM because of the presence of the oscillations caused by Brillouin scattering. However, our calculations of the diffraction signal only due to surface displacement (blue curve) suggests that acoustic dispersion plays a role. No measurable diffraction by the acoustic wave propagating in $\mathrm{Ni}$ is observed after $450 \mathrm{ps}$. The main reason for this is that the acoustic wave undergoes damping as it propagates inside the Ni layer and because of acoustic reflection losses at the glass-Ni interface upon every round trip.

\section{B. Detection of grating under metallic and multiple dielectric layers}

Now that we understand the experiments performed on buried gratings deposited on single $\mathrm{Au}$ and Ni layers, we next perform measurements on more complex samples with multiple dielectric layers. We fabricate samples that consist of layers of materials that partially mimic the materials and structure used in the fabrication of 3D NAND memory [38]. For the first sample, which will henceforth be referred to as the "Au-multilayer" sample, we evaporate $145 \mathrm{~nm}$ of $\mathrm{Au}$ on glass, on top of which we deposit five alternating pairs of 18 -nm-thick $\mathrm{SiO}_{2}$ and 18-nm-thick $\mathrm{Si}_{3} \mathrm{~N}_{4}$. On top of this stack, we fabricate a $10-\mathrm{nm}$ amplitude Au grating, as shown in Fig. 4(a). For the second sample, which is henceforth referred to as the "Ni-multilayer" sample, we first deposit a 147-nm-thick layer of $\mathrm{Ni}$ followed by the deposition of ten pairs of 18-nm-thick $\mathrm{SiO}_{2}$ and 18-nmthick $\mathrm{Si}_{3} \mathrm{~N}_{4}$ layers. On top of this, a $10-\mathrm{nm}$ amplitude $\mathrm{Au}$ grating is fabricated. Both gratings have a pitch of $6 \mu \mathrm{m}$ and a $50 \%$ duty cycle. In this case, the $\mathrm{SiO}_{2} / \mathrm{Si}_{3} \mathrm{~N}_{4}$ stack consists of 20 layers in total and is thus twice as thick as the stack in the first sample. This sample is schematically shown in Fig. 4(b). The thickness of the $\mathrm{SiO}_{2}$ and $\mathrm{Si}_{3} \mathrm{~N}_{4}$ layers is calibrated by linear spectroscopy measurements. Details about the sample fabrication are provided in Appendix C.

In Fig. 4(c) we show the measured diffraction signal as a function of the pump-probe delay (red curve), for the " $\mathrm{Au}$ multilayer" sample. The diffraction signal remains zero for about $100 \mathrm{ps}$, after which the first diffraction peak is observed, followed by a quasiperiodic oscillating signal. This means that, in spite of the many interfaces encountered by the propagating acoustic wave, a well-defined acoustic copy of the buried grating can still be detected near the glass-Au interface. Apparently, the energy of the acoustic wave has not completely dissipated as it propagates through these layers. Similar to the measurements on the grating fabricated on a single layer of $\mathrm{Au}$, the individual peaks are fairly broad. Again, this is caused by the relative homogeneous heating of the 145-nm-thick $\mathrm{Au}$ layer by rapid diffusion of the electron-gas energy into the metal layer. As a result the longitudinal acoustic pulse has a wavelength corresponding to twice the thickness of the $\mathrm{Au}$ layer. The diffraction signal we observe is the result of a grating-shaped displacement of the glass-Au interface and also due to Brillouin scattering in the glass substrate.

We perform numerical simulations to show how these factors affect the shape and time evolution of the diffraction signal. The numerically calculated diffraction signal versus time delay for the "Au-multilayer" sample, is shown in Fig. 4(c), upper panel (black curve). We see a remarkable agreement between the measured and the simulated curves for this complex multilayer sample, indicating that the model contains all the physics necessary to predict the salient features of our measurements. The bottom panel in the same figure shows the contributions to the calculated diffraction signal due to the (i) displacement of the atoms near the glass-metal interface caused by the acoustic wave propagating in the Au layer (blue curve) and due to (ii) changes in the optical constants of the glass due to the strain-optic effect, or Brillouin scattering, in the glass substrate (green curve). For the "Au-multilayer" sample, the first peak for the displacement contribution (blue curve) is at $146 \mathrm{ps}$, which corresponds to the acoustic round trip time for the entire sample. Note that only acoustic waves that have reflected off the buried grating give rise to optical diffraction. Earlier reflections of the acoustic waves from various interfaces it encounters as the wave propagates towards the buried grating for the first time are thus not detected. The videos showing the calculated time-dependent strain and displacement inside the "Au-multilayer" sample are available as Videos 1 and 2, respectively. The description of the videos can be found in Appendix D.

While it is tempting to try to attribute the timing of various peaks in the diffraction signal to the propagation time of acoustic waves and reflection of those acoustic waves inside the sample, in reality, this is difficult. Moreover, predicting the strength of the diffraction peaks is impossible without resorting to numerical calculations that take all possible contributions to the diffraction into account, as required for high-precision wafer-alignment applications. A case in point is the diffracted signal shown in Fig. 4(c). Whereas one would intuitively expect that the first acoustic reflection of the grating to reach the glass-Au interface (at $146 \mathrm{ps}$ ) will provide the strongest diffracted signal, the measurement clearly shows that this is not the case. Instead, the second diffraction peak at $197 \mathrm{ps}$ is 

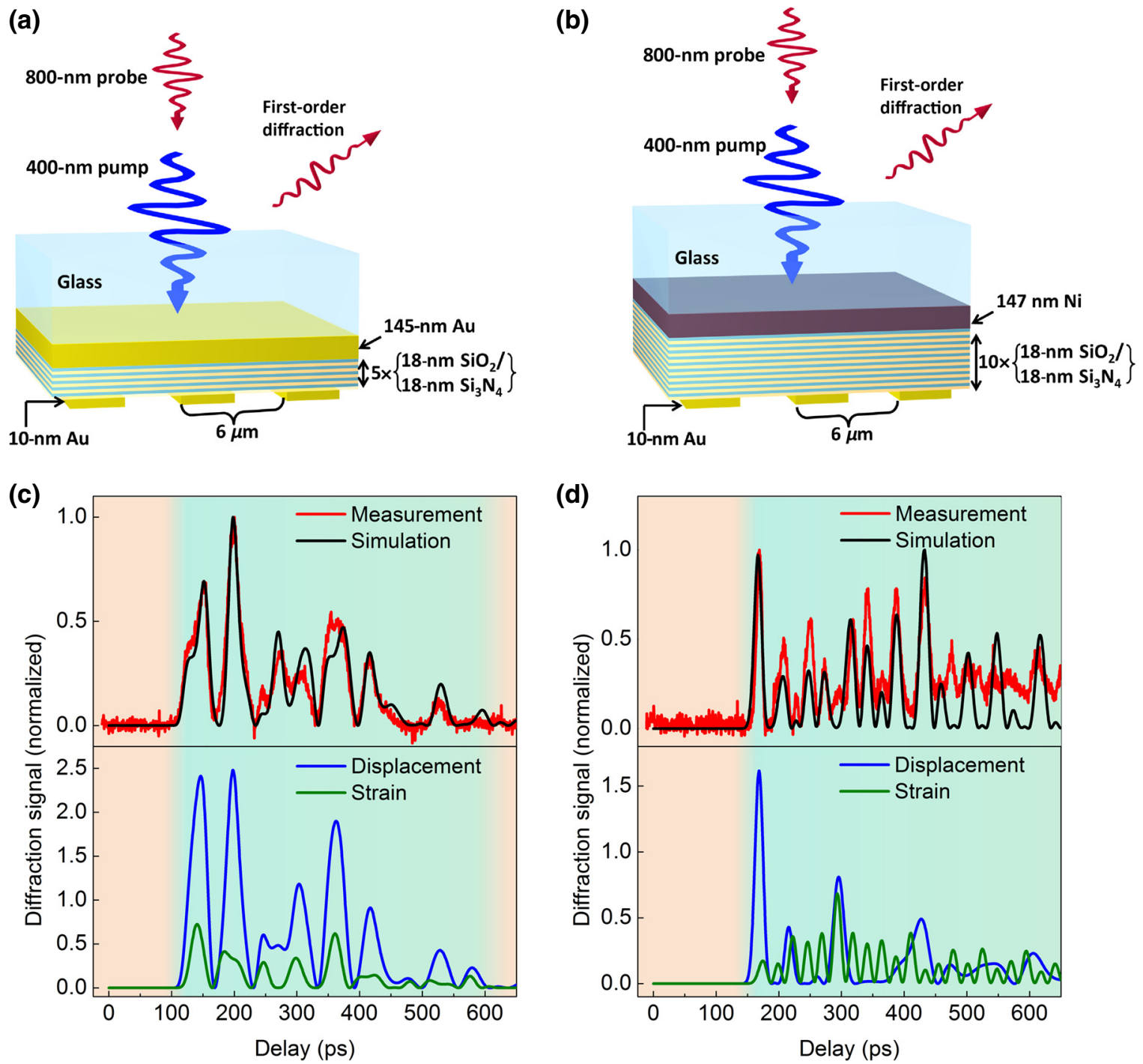

FIG. 4. Schematics of the beam and sample geometry for (a) the 10-nm amplitude Au grating on five pairs of alternating layers of 18-nm $\mathrm{SiO}_{2}$ and 18-nm $\mathrm{Si}_{3} \mathrm{~N}_{4}$ fabricated on a 145-nm-thick Au layer on glass ("Au-multilayer"), (b) the 10-nm amplitude Au grating on ten pairs of alternating layers of 18-nm $\mathrm{SiO}_{2}$ and 18-nm $\mathrm{Si}_{3} \mathrm{~N}_{4}$, fabricated on top of a 147-nm-thick Ni layer on glass ("Ni-multilayer"). (c) Upper panel: experimentally measured (red) and numerically simulated (black) diffracted probe signal versus pump-probe delay for the "Au-multilayer" sample. Bottom panel: calculated probe diffraction signal versus pump-probe delay taking only the displacement of the glass-Au interface into account (blue line), and taking only the propagating strain pulse in the glass substrate into account (green line). (d) Upper panel: experimentally measured (red) and numerically simulated (black) diffracted probe signal versus pump-probe delay for the "Ni-multilayer" sample. Bottom panel: calculated probe diffraction signal verus pump-probe delay taking only the displacement of the glass-Ni interface into account (blue line), and taking only the propagating strain pulse in the glass substrate into account (green line).

clearly stronger than the first diffraction peak (black curve). The diffracted signals due to surface displacement (blue curve) have similar strengths at 146 and $197 \mathrm{ps,} \mathrm{while} \mathrm{the}$ diffracted signals due to Brillouin scattering in the glass (green curve) have different strengths. The total diffracted signals, both at 146 and $197 \mathrm{ps}$ are the result of destructive interference between the diffracted fields (due to interface displacement and Brillouin scattering). The lower strength of the diffracted signal from Brillouin scattering at $197 \mathrm{ps,}$ results in the total diffracted signal at 197 ps being higher than that at 146 ps. Later, in Sec. III D, we also show that the interface displacement gratings at 146 and 197 ps are inverted with respect to each other.

In Fig. 4(d) we plot the measured diffraction signal as a function of the pump-probe delay (red curve), for the "Ni-multilayer" sample. The diffraction signal remains zero for about $155 \mathrm{ps}$, then begins to increase until a first maximum is observed at $169 \pm 2$ ps. This diffraction signal is due to the acoustic wave returning to the glass-Ni interface after one round trip through the whole stack of 
layers. This arrival time matches the expected propagation time through all the layers (see Table I). The diffraction signal then drops to zero and quasiperiodic oscillations are observed. Note that here too the diffraction peaks are "sharper" than for the "Au-multilayer" sample, in a manner similar to what is observed for the single-layer samples.

In $\mathrm{Ni}$, the hot electron gas formed after optical excitation cools much more rapidly and heats the lattice before the hot-electron-gas energy can diffuse much deeper into the layer. The resultant acoustic wave is much more localized in the propagation direction than in the case of $\mathrm{Au}$ and thus gives rise to more narrow peaks in the diffraction signals. The signal we observe after 169 ps has contributions from the interference of optical fields diffracted off acoustic waves near the glass-Ni interface, and off acoustic waves transmitted into the glass substrate.

To better understand the measurement, we perform a numerical simulation of the diffracted signal, which is shown in Fig. 4(d), (black curve). The position of the peaks in the simulated curve agrees well with the position of the peaks in the measurement. The amplitudes are seen to match less well. The blue curve in the bottom panel in the figure shows the calculated diffracted intensity taking only the displacement of the glass-Ni interface into account, and the green curve shows the diffracted intensity calculated taking only the Brillouin scattering in glass into account. The first peak seen in the calculation of the diffracted signal caused by only the displacement, at $169 \mathrm{ps}$, is due to the return of the first acoustic wave reflected off the buried grating after propagating through all the layers. This grating-shaped acoustic wave, now at the glass-Ni interface, undergoes another reflection inside the $147-\mathrm{nm}$ Ni layer before it returns to the glass-Ni interface again where it gives rise to the second peak at $215 \mathrm{ps}$. The periodic oscillations due to Brillouin scattering in the glass substrate can be seen more clearly in the "Nimultilayer" sample as compared to the "Au-multilayer" sample, because of the shorter length of the acoustic wave. Our measurement on the "Ni-multilayer" sample demonstrates that we can detect the acoustic wave even after it has propagated back and forth through 20 dielectric layers, so through 40 layers in total.

Finally, we note that shortly after optical excitation with a pump beam, the abrupt expansion of the metal layer also generates an acoustic wave, which propagates backwards into the glass. However, this acoustic wave does not result in the diffraction of the probe pulse, as its wavefront has not reflected off the grating and is, thus, "flat."

\section{Optical excitation of Au and Ni layers}

To gain some insight into the longitudinal spatial extent of the acoustic wave generated after optical excitation, we use the two-temperature model (TTM) to calculate the lattice temperature as a function of depth inside the metal
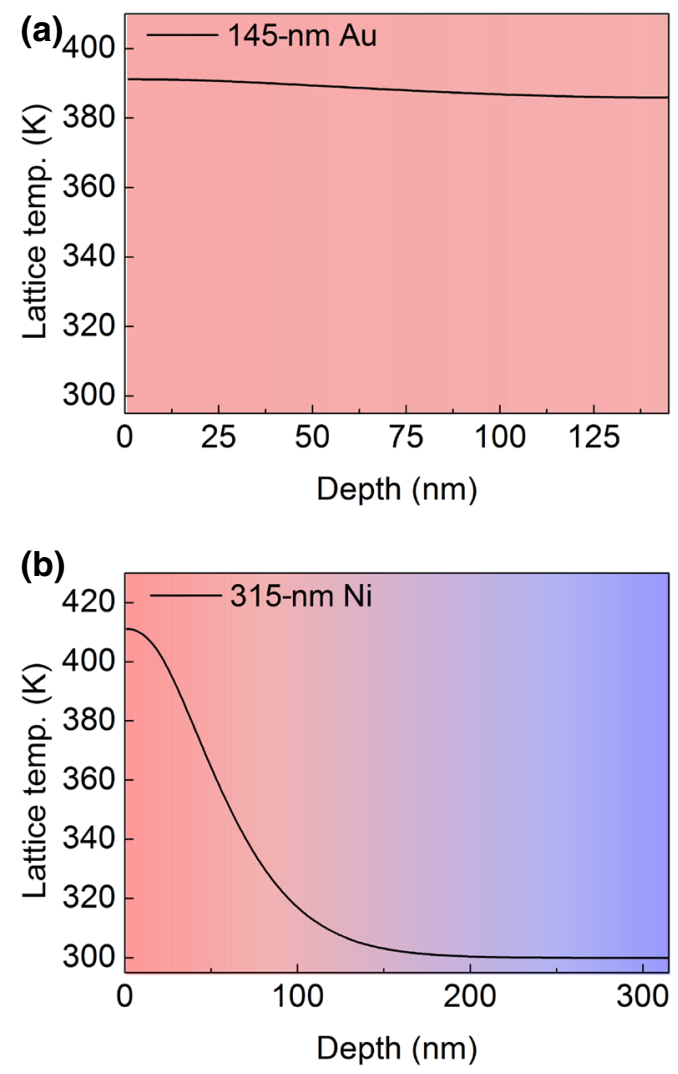

FIG. 5. Lattice temperature inside the metal as a function of depth in the metal 15 ps after optical excitation with a $400-\mathrm{nm}$ pump pulse for (a) a 145-nm Au layer and (b) a 147-nm Ni layer.

layer, 15 ps after optical excitation with the pump pulse. At this time the TTM shows that the hot electron gas has significantly cooled and is in local thermal equilibrium with the lattice. In Fig. 5(a), we show the resulting lattice temperature as a function of depth inside the 145$\mathrm{nm}$-thick $\mathrm{Au}$ layer present in between the glass and five pairs of $\mathrm{SiO}_{2} / \mathrm{Si}_{3} \mathrm{~N}_{4}$. The figure shows the large penetration depth of energy into the metal, which can be explained by the relatively weak electron-phonon coupling strength of $\mathrm{Au}$. The Au layer is thin enough that after $15 \mathrm{ps}$, the lattice is heated almost homogeneously. There is practically no spatial gradient in the lattice temperature. In Fig. 5(b), we plot the calculated lattice temperature as a function of depth for the 147-nm-thick Ni layer, on which the ten pairs of $\mathrm{SiO}_{2}$ and $\mathrm{Si}_{3} \mathrm{~N}_{4}$ layers are fabricated. In contrast to the calculations for 145-nm Au, here the lattice temperature distribution still shows a significant temperature gradient. The acoustic wave launched by the heated lattice in this case is, therefore, shorter than that in the Au layer.

\section{Displacement amplitude at the glass-metal interface}

To get an estimate of the typical surface displacement amplitudes near the glass-metal interface, we plot in Fig. 6 the lattice displacement as a function of the position along 

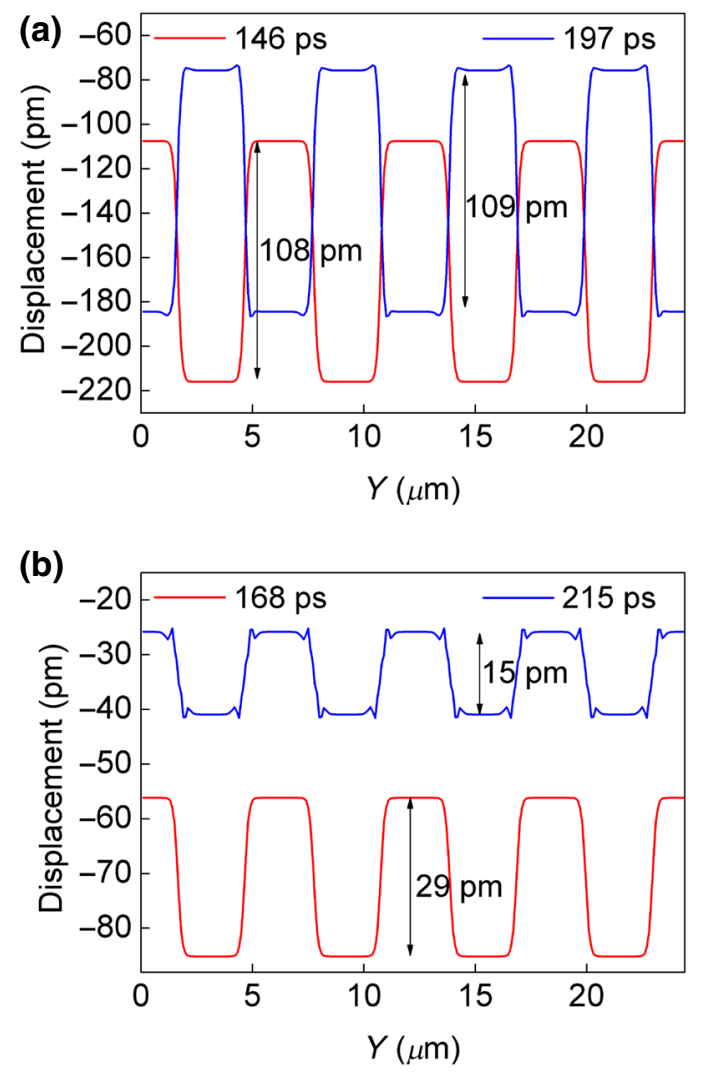

FIG. 6. (a) Lattice displacement calculated at the glass-Au interface for the "Au-multilayer" sample at time delays of 146 and 197 ps after optical excitation. (b) Lattice displacement calculated at the glass-Ni interface for the "Ni-multilayer" sample at time delays of 168 and 215 ps after optical excitation.

the direction perpendicular to the grating lines for two different times after optical excitation, for the four different samples discussed in this paper. Here, four unit cells used in the simulations are shown for clarity, where one unit cell has a width of $6 \mu \mathrm{m}$. In the convention used here, the unperturbed glass-metal interface has zero amplitude displacement, and a negative displacement implies a movement of the interface in the direction of the glass substrate. In Fig. 6(a), we show the displacement at the glass-Au interface for the "Au-multilayer" sample at pump-probe delays of 146 ps (red curve) and 197 ps (blue curve). The grating-shaped displacement at $146 \mathrm{ps}$ has the same phase as the buried grating and has a peak-to-valley amplitude of $108 \mathrm{pm}$. The lattice displacement shows an offset of over $100 \mathrm{pm}$, which is partially caused by the acoustic wave and partially by the expansion of the lattice due to heating. However, only the grating-shaped displacement profile contributes to the diffraction efficiency.

For industrial wafer-alignment applications it is essential to predict the exact position of the maximum signal, and the sensor should be optimized to measure the strongest signal possible. More importantly, we should know the phase of the acoustic grating, that is, whether the acoustic grating is inverted with respect to that of the buried grating or not. This is because wafer alignment in the semiconductor industry relies on measuring the optical phase of the diffracted light, which is dependent on the spatial phase of the grating. In Fig. 6(a) the interface grating at 146 ps retains the spatial phase of the buried grating, while the grating at $197 \mathrm{ps}$ is inverted. This is due to interference of multiple acoustic waves inside the sample created by the partial reflection and transmission of the waves at the $\mathrm{Au}-$ dielectric interface. Since the diffracted light at both 146 and $197 \mathrm{ps}$ is dominated by the light diffracted from the interface displacement, the inversion of the grating means that the diffracted light at $197 \mathrm{ps}$ is $\pi$ out of phase with the diffracted light at $146 \mathrm{ps}$.

In Fig. 6(b) we show the displacement at the glass-Ni interface for the "Ni-multilayer" at a pump-probe delay of $168 \mathrm{ps}$, when the acoustic wave has completed one round trip (red curve). The grating-shaped displacement profile has a peak-to-valley amplitude of $29 \mathrm{pm}$ and has the same phase as that of the buried grating. The grating-shaped acoustic wave, now at the glass-Ni interface, undergoes another reflection inside the 147-nm Ni layer. The acoustic wave returns to the glass-Ni interface and results in optical diffraction 215 ps after optical excitation. Hence, the grating-shaped displacement at $215 \mathrm{ps}$ [blue curve in Fig. 6(b)], has the same phase as the one at 168 ps but a reduced peak-to-valley amplitude of $15 \mathrm{pm}$. This displacement grating has a lower amplitude because the acoustic wave is also transmitted into the stack of dielectric layers upon reflection at the $\mathrm{Ni}-\mathrm{SiO}_{2} / \mathrm{Si}_{3} \mathrm{~N}_{4}$ interface, and also due to damping and dispersion of the acoustic wave during the propagation through the $147-\mathrm{nm}$ Ni layer.

\section{E. Effective acoustic properties of the bilayer dielectric stack}

To understand how the $\mathrm{SiO}_{2}$ and $\mathrm{Si}_{3} \mathrm{~N}_{4}$ bilayers affect the strength and shape of the diffraction signal, we perform numerical simulations where we replace the dielectric bilayers with a single equivalent acoustic medium [49]. We replace the dielectric layers with a single medium that has the same thickness as the $\mathrm{SiO}_{2} / \mathrm{Si}_{3} \mathrm{~N}_{4}$ stack and has a density that is the average density of $\mathrm{SiO}_{2}$ and $\mathrm{Si}_{3} \mathrm{~N}_{4}$. The acoustic velocity of the equivalent medium is calculated such that the time for the acoustic wave to propagate through a single equivalent medium is the same as the time required to propagate through all the dielectric layers. Hence the velocity of the equivalent time-average medium $\left(V_{\mathrm{TA}}\right)$ is given by

$$
\frac{1}{V_{\mathrm{TA}}}=\frac{1}{d_{1}+d_{2}}\left(\frac{d_{1}}{v_{1}}+\frac{d_{2}}{v_{2}}\right),
$$

where $v_{1}$ and $v_{2}$ are the acoustic velocities in $\mathrm{SiO}_{2}$ and $\mathrm{Si}_{3} \mathrm{~N}_{4}$, respectively, and $d_{1}$ and $d_{2}$ are the thickness of 
(a)

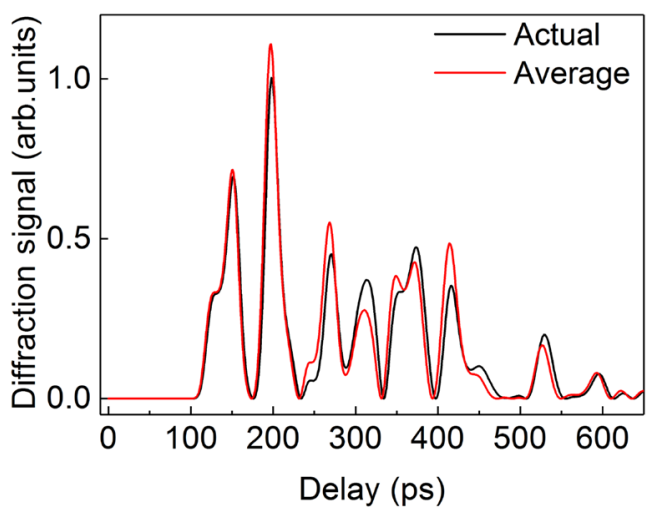

(b)

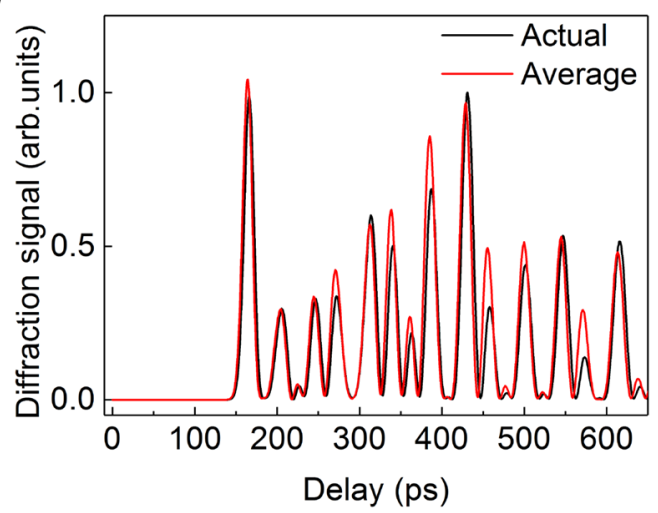

FIG. 7. (a) Calculated diffraction signal versus pump-probe delay for the "Au-multilayer" sample. The black curve is a calculation that takes all pairs of alternating layers of $18-\mathrm{nm} \mathrm{SiO}_{2}$ and $18-\mathrm{nm} \mathrm{Si}_{3} \mathrm{~N}_{4}$ into account. The red curve is a calculation in which all pairs of alternating layers are replaced with a single layer having the same total thickness but with average acoustic properties. (b) Similar to (a) but now for the "Ni-multilayer" sample.

$\mathrm{SiO}_{2}$ and $\mathrm{Si}_{3} \mathrm{~N}_{4}$, respectively. In Fig. 7(a) we show the calculated time-dependent diffraction signal for the "Aumultilayer" sample (black curve), together with the diffraction signal calculated when the ten dielectric layers are replaced with the single equivalent time-average medium of the same thickness (red curve). Similarly, in Fig. 7(b) we show the calculated time-dependent diffraction signal for the "Ni-multilayer" sample (black curve) and the calculated diffraction signal when the 20 dielectric layers are replaced with the single equivalent medium of the same thickness (red curve). For both samples, although there are some differences between the calculated curves, the signal shapes are remarkably similar, as are the amplitudes of the signals. Minor differences are mostly seen at long time delays. The position of the first acoustic diffraction peak is not expected to change when the bilayer is replaced with an equivalent acoustic medium because the time it takes for one round trip inside the whole layer remains the same.

To elucidate the effect that both acoustic impedance mismatch and layer thickness have on the strength of the first acoustic signals, we first perform a calculation in which we ignore the dielectric layer thickness and only calculate the strength of an acoustic signal after propagating through $38 \mathrm{SiO}_{2} / \mathrm{Si}_{3} \mathrm{~N}_{4}$ interfaces. Using the classical acoustic wave equations, we calculate the intensity of the acoustic wave transmitted through one $\mathrm{SiO}_{2} / \mathrm{Si}_{3} \mathrm{~N}_{4}$ interface $A_{T}$ as

$$
A_{T} / A_{I}=\frac{4 Z_{1} Z_{2}}{\left(Z_{1}+Z_{2}\right)^{2}},
$$

where $A_{I}$ is the intensity of the incident acoustic wave and $Z_{1}$ and $Z_{2}$ are the acoustic impedance of $\mathrm{SiO}_{2}$ and $\mathrm{Si}_{3} \mathrm{~N}_{4}$ layer, respectively. We calculate the transmission coefficient $\left(A_{T} / A_{I}\right)$ to be 0.982 . Since there are 20 dielectric layers in the "Ni-multilayer" sample, the first acoustic echo has to travel through $2 \times 19$ dielectric interfaces, apart from the Ni-dielectric stack interface. This means that after propagating back and forth through the 20 dielectric layers, the intensity of the acoustic wave should be reduced by $(0.982)^{38}=0.5014$ in comparison to a case where the dielectrics are replaced with a single equivalent medium. This shows that, in principle, the modest acoustic impedance mismatch between the $\mathrm{SiO}_{2}$ and $\mathrm{Si}_{3} \mathrm{~N}_{4}$ can give rise to significant amplitude changes after propagating through 38 interfaces. The difference between this simple calculation and the full numerical one is, of course, that interference between the multiple acoustic waves reflected at various interfaces in these very thin layers is not taken into account. In spite of this, we find that in the numerical simulations the red and black curves are very similar, and therefore, the bilayer interfaces do not change the shape and strength of the time-dependent diffraction signal significantly. This is partially explained by the length of the acoustic wave, which is much greater than the thickness of the individual dielectric layers. The acoustic wave only "sees" an equivalent medium rather than the individual layers. However, the modest acoustic impedance mismatch between the $\mathrm{SiO}_{2}$ and $\mathrm{Si}_{3} \mathrm{~N}_{4}$ also plays a role here (see Table I), and both effects must therefore be taken into account to understand these results. In Appendix E, we show a similar calculation on a hypothetical sample similar to that of the "Ni-multilayer" sample, where the thicknesses of the dielectric layers $\left(\mathrm{SiO}_{2}\right.$ and $\left.\mathrm{Si}_{3} \mathrm{~N}_{4}\right)$ are increased to $200 \mathrm{~nm}$. There we see that the strength of the first echo after propagating through multiple dielectric interfaces is considerably weaker when compared to a case where the dielectric layers are replaced with a timeaveraged medium of the same thickness. This means that, when the thickness of the dielectric layers is larger than the acoustic wavelength, the dielectric interfaces have a significant effect on the propagating acoustic waves, despite the modest acoustic impedance mismatch.

Similar effects have been predicted for the propagation of low-frequency sound waves in seismology [49] and we 
TABLE I. Acoustic properties of different materials used in our calculations $[9,26]$.

\begin{tabular}{lcc}
\hline \hline & $\begin{array}{c}\text { Sound velocity } \\
(\mathrm{m} / \mathrm{s})\end{array}$ & $\begin{array}{c}\text { Acoustic impedance } \\
\left(10^{6} \mathrm{Ns} / \mathrm{m}^{3}\right)\end{array}$ \\
\hline $\mathrm{Au}$ & 3200 & 61.8 \\
$\mathrm{Ni}$ & 5800 & 51.5 \\
$\mathrm{Glass} \mathrm{substrate}$ & 5700 & 12.5 \\
$\mathrm{SiO}_{2}$ & 5100 & 13.5 \\
$\mathrm{Si}_{3} \mathrm{~N}_{4}$ & 5600 & 17.8 \\
\hline \hline
\end{tabular}

show that it holds true also for such high-frequency acoustic waves. This suggests that laser-induced ultrasonics can be used to detect buried gratings when the number of thin dielectric layers is further increased to values $(>100)$ often encountered in the semiconductor manufacturing industry.

\section{MATERIAL PROPERTIES}

In Table I we list the acoustic properties used in the numerical simulations.

\section{CONCLUSION}

We show that laser-induced ultrasonics can be used to detect the presence of gratings buried under optically opaque metal and dielectric layers. We observe optical diffraction from the acoustic wave reflected from a grating buried under thick metal and multiple dielectric layers. The diffraction is due to the grating-shaped displacement of the glass-metal interface and Brillouin scattering in the glass substrate. The total diffracted signal measured is the coherent sum of the electric fields diffracted from the acoustic grating at the glass-metal interface and the acoustic grating in the glass. Our experiments are in very good agreement with calculations using a numerical model that takes all these effects into account. Comparing the numerical calculations with the experimental results allows us to, for example, conclude that some acoustic-wave-induced gratings at the glass-metal interface are inverted with respect to the buried grating. It also allows us to understand and predict the strength of diffraction peaks strongly affected by destructive or constructive interference between the optical fields diffracted by the multiple acoustic copies inside the metal and inside the glass. The ability to understand and predict the time-dependent shape and origin of all peaks in the diffraction signal in this way is crucial for applications of laser-induced ultrasound for wafer alignment in the semiconductor manufacturing industry.

\section{ACKNOWLEDGMENTS}

This work is carried out at the Advanced Research Center for Nanolithography (ARCNL), a public-private partnership of the University of Amsterdam (UvA), the Vrije
Universiteit Amsterdam (VU), the Netherlands Organisation for Scientific Research (NWO), and the semiconductor equipment manufacturer ASML.

\section{APPENDIX A: DETECTION OF HIDDEN GRATING USING ULTRAFAST ELECTRON DYNAMICS}

In Ref. [40], we showed that hidden gratings can be detected underneath a 100 -nm-thick gold layer, by optical measurements of ultrafast electron dynamics. In that experiment, diffraction signals were measured due to an electron temperature grating that forms after exciting a flat gold layer (covering a grating) with a pump pulse. The temperature grating is the result of the different electrongas dynamics above the valleys and the peaks of the buried grating. Such a temperature grating leads to a grating in the optical constants near the surface of the gold from which a probe pulse can be diffracted, thus proving the presence of the buried grating. However, diffraction due to electron dynamics can only be observed when the grating is buried underneath a single thin metal layer (approximately 100 $\mathrm{nm}$ ), and only when the metal has a low electron-phonon coupling strength (such as is the case for $\mathrm{Au}$ and $\mathrm{Ag}$ ).

The scope of the experiments described in the present paper is much broader. Using laser-induced ultrasonics we can detect buried gratings, (i) underneath thick metal layers (shown by measurements on the 522-nm Au sample and 315-nm Ni sample), (ii) underneath dielectric layers (shown by measurements on the "Au/Ni-multilayer" samples), (iii) underneath metals with high electron-phonon coupling strength (shown by measurements on the 315-nm Ni sample and "Ni-multilayer" sample). Ultrafast electron dynamics cannot be used to detect gratings underneath the complex samples discussed in this manuscript. This is due to the inability of the hot electron gas to penetrate the dielectric layers. Indeed, no diffraction signals due to electron dynamics can be seen in the measurements discussed in the paper.

\section{APPENDIX B: NUMERICAL SIMULATIONS}

An advanced 2D numerical model, which captures the generation, propagation, and detection of high-frequency acoustic waves by ultrafast laser pulses, is used to simulate the diffracted signal [39]. The model consists of three main parts, (i) absorption of light and the subsequent generation of the acoustic wave, (ii) propagation of the acoustic wave, (iii) detection of the acoustic wave. The absorption of the femtosecond laser pulse and the subsequent heating and cooling of the electron gas inside a metal layer is described by the well-known TTM [41,50-54]. The heating of the lattice calculated from the TTM sets up an isotropic thermal stress, which leads to the generation of the high-frequency acoustic wave. The equation of motion for an isotropic, linear elastic wave is used to describe 
TABLE II. Properties of the $\mathrm{Au}$ and Ni used in our TTM calculations $[41-43,55,56]$.

\begin{tabular}{lcc}
\hline \hline & $\mathrm{Au}$ & $\mathrm{Ni}$ \\
\hline Optical penetration depth at $400 \mathrm{~nm}(\mathrm{~nm})$ & 16 & 12 \\
Optical penetration depth at $800 \mathrm{~nm}(\mathrm{~nm})$ & 13 & 13 \\
Electron-phonon coupling constant & 3.2 & 36 \\
$\left(10^{16} \mathrm{Wm}^{-3} \mathrm{~K}^{-1}\right)$ & & \\
\hline \hline
\end{tabular}

the propagation of the acoustic wave inside the metal and dielectric layers. Finally, by propagating the complex electric field of the probe pulse after the optical excitation by the pump pulse, through the substrate and into the metal, we can calculate the diffraction efficiency as a function of time delay. The model calculates the first-order diffracted signal by accounting for the spatially periodic changes in refractive index due to the thermo-optic effect and the strain-optic effect, and the spatially periodic displacement of the glass-metal interface.

In Table II we list the optical properties used in the numerical simulations.

\section{APPENDIX C: SAMPLE FABRICATION}

In principle, buried gratings can be made by chemically etching gratings in $\mathrm{Si}$, followed by the deposition of dielectric and metallic layers. The resulting sample, however, then shows a strong surface topography, which follows the topography of the buried grating even though it is not a true copy of it. When real semiconductor devices are manufactured, repeated steps involving deposition of layers followed by polishing are carried out, ultimately reducing or eliminating the residual surface topography. Making samples with zero surface topography turned out to be impossible using our clean-room facilities. To test whether laser-induced ultrasonics is capable of detecting buried gratings, we therefore opt to make samples by first depositing nominally flat dielectric and metallic layers on glass followed by the fabrication of a grating on top of this. By now performing pump-probe diffraction measurements from the glass side, the grating is invisible to both pump and probe and can be viewed as a buried grating. All the samples are prepared on 175- $\mu$ m-thick, chemically cleaned glass substrates. The Au and Ni layers are fabricated by physical vapor deposition, and the thickness is determined by a quartz crystal thickness monitor. The gratings on top of the metal and dielectric layers are fabricated by UV optical lithography. All gratings used in this research have a pitch of $6 \mu \mathrm{m}$. The $\mathrm{SiO}_{2}$ and $\mathrm{Si}_{3} \mathrm{~N}_{4}$ layers are deposited by sputtering, using a silicon target in the presence of oxygen and nitrogen, respectively. To calibrate the thickness of the $\mathrm{SiO}_{2}$ and $\mathrm{Si}_{3} \mathrm{~N}_{4}$ layers, we perform linear spectroscopy measurements on single layers of $\mathrm{SiO}_{2}$, and $\mathrm{Si}_{3} \mathrm{~N}_{4}$, deposited on $\mathrm{Si}$ under the same conditions as the multilayer structures.

\section{APPENDIX D: VIDEOS OF STRAIN AND DISPLACEMENT DYNAMICS}

The propagation of the strain inside the "Au-multilayer" sample calculated using the numerical simulations can be seen in Video 1. On the left side of the video is the schematic of the sample and on the right is the video of the time-dependent strain inside the whole sample. The $y$ axis is the position along the direction perpendicular to the grating lines. The color scheme shows the strain, and the time is displayed on the top of the video. Here, four unit cells used in the simulations are shown for clarity, where one unit cell has a width of $66 \mu \mathrm{m}$. The thickness of the glass substrate used in the measurements is $175 \mu \mathrm{m}$, and in the simulations we use a $6-\mu \mathrm{m}$-thick layer to model the glass substrate. A 6- $\mu$ m-thick layer is sufficient for modeling as the acoustic wave does not propagate more than $5 \mu \mathrm{m}$ during the time duration of the measurements. However, for clarity only $0.85 \mu \mathrm{m}$ of the glass is shown in the video. We also show the displacement at different positions as a function of time inside the "Au-multilayer" sample in Video 2. This video is similar to Video 1, except the color scheme represents the displacement amplitude inside the sample.

All the physical effects discussed in this paper can be seen in these videos. The uniform heating of the 145-nmthick Au layer launches an acoustic wave, which exerts strain at both ends of the 145-nm Au layer (Video 1). The strain wave propagates through the $\mathrm{Au}$ and dielectric layers, reflects off the buried, and acquires a grating shape. The grating-shaped strain wave can be seen in the glass, as it moves away from the glass-Au interface. The Brillouin scattering we observe in the measurements is due to this grating-shaped strain wave in the glass. Note that the strength of the strain wave appears different in $\mathrm{Au}$, glass, $\mathrm{SiO}_{2}$, and $\mathrm{Si}_{3} \mathrm{~N}_{4}$ because strain depends on the mechanical properties of the material. Multiple partial reflections and transmissions of the strain wave at the glass- $\mathrm{Au}$ and $\mathrm{Au}-$ dielectric stack interface can also be seen in the video. At longer time delays the strain wave decays due to damping, dispersion, and losses upon partial reflection and transmission at the interfaces. In Video 2, we can also see the displacement amplitudes inside the sample due to the generation, propagation, and reflection of the acoustic wave.

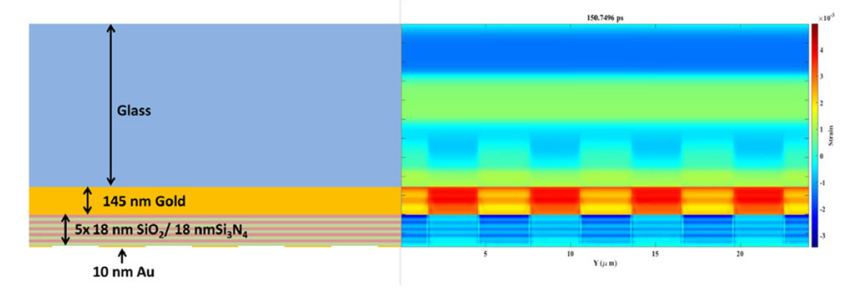

VIDEO 1. Propagation of strain inside the "Au-multilayer" sample. 


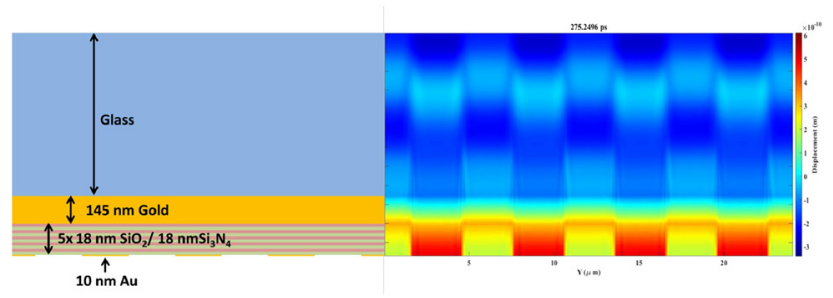

VIDEO 2. Calculated displacement amplitudes inside the "Au-multilayer" sample.

Here we can see the grating-shaped displacement at the glass-Au interface after the acoustic wave has reflected off the buried grating, as well as changes in the spatial phase of the displacement grating by $\pi$ between 146 and 197 ps.

\section{APPENDIX E: EFFECTIVE ACOUSTIC PROPERTIES OF THE BILAYER DIELECTRIC STACK ON A HYPOTHETICAL SAMPLE}

In this section, we show that the dielectric layer stack does not behave as a time-averaged equivalent medium when the wavelength of the acoustic wave is smaller than the thickness of the individual layers, despite the modest acoustic impedance mismatch between the layers. We perform calculations similar to what we show in Sec. E in the main paper but now on a hypothetical sample. The hypothetical sample is similar to the "Ni-multilayer" sample, while the only difference is that the thicknesses of individual dielectric layers $\left(\mathrm{SiO}_{2}\right.$ and $\left.\mathrm{Si}_{3} \mathrm{~N}_{4}\right)$ are increased to $200 \mathrm{~nm}$. The blue curve in Fig. 8 is the calculated timedependent diffraction signal for this hypothetical sample,

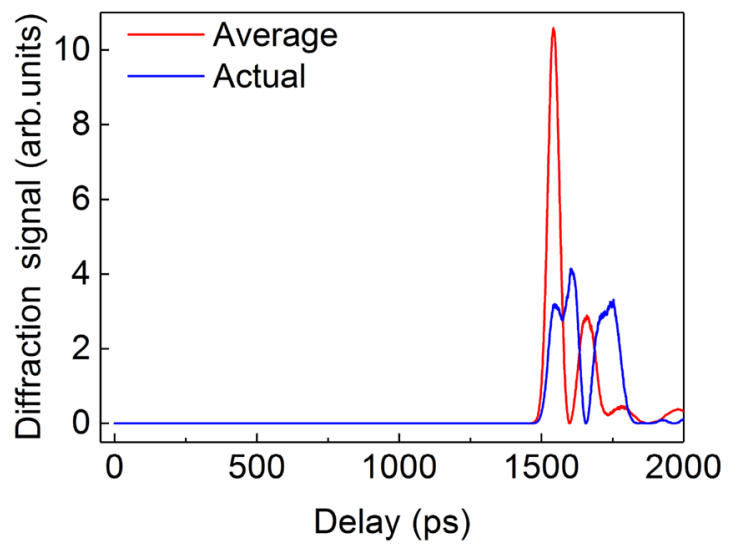

FIG. 8. Calculated diffraction signal versus pump-probe delay for a hypothetical sample similar to the "Ni-multilayer" sample, but the thicknesses of dielectric layers are increased to $200 \mathrm{~nm}$. The black curve is a calculation that takes all pairs of alternating layers of 200-nm $\mathrm{SiO}_{2}$ and 200-nm $\mathrm{Si}_{3} \mathrm{~N}_{4}$ into account. The red curve is a calculation in which all pairs of alternating layers have been replaced with a single layer having the same total thickness but with average acoustic properties. and the red curve is the calculated diffraction signal when the 20 dielectric layers are replaced with the single equivalent medium of the same thickness $(20 \times 200 \mathrm{~nm})$. As expected, the position of the first diffraction peak is exactly the same for both curves. However, the intensity of the first blue peak is much weaker than that of the first red peak. This is in contrast to what we observed in the case of the actual "Ni-multilayer" sample, where the strength of the first diffraction peaks is very similar (Fig. 7 in the main paper). This difference between the two samples is observe in spite of the fact that the "Ni-multilayer" sample and the hypothetical sample have the exact same number of interfaces and the same acoustic properties. For a sample with thick dielectric layers, therefore, the effect of a modest acoustic impedance mismatch between the $\mathrm{SiO}_{2}$ and $\mathrm{Si}_{3} \mathrm{~N}_{4}$ on the transmitted acoustic wave is much larger for a sample with a thin layer. In the latter case, the stack of layers behaves more like a single "equivalent" medium.

This shows that for a stack of dielectric layers to behave as a single acoustic medium, the wavelength of the acoustic wave should be comparable to that of the individual layer.

[1] A. Nolvi, I. Kassamakov, and E. Hæggström, Subsurface metrology using scanning white light interferometry: Absolute $\mathrm{z}$ coordinates deep inside displays, J. Opt. Soc. Am. A 35, A18 (2018).

[2] M. H. van Es, A. Mohtashami, D. Piras, and H. Sadeghian, Image-based overlay and alignment metrology through optically opaque media with sub-surface probe microscopy, Proc. SPIE 10585, 28 (2018).

[3] R. van Gastel, G. Hlawacek, H. J. Zandvliet, and B. Poelsema, Subsurface analysis of semiconductor structures with helium ion microscopy, Microelectron. Reliab. 52, 2104 (2012).

[4] A. C. Rudack, L. W. Kong, and G. G. Baker, in IEEE/SEMI Advanced Semiconductor Manufacturing Conference (ASMC, San Jose, 2010), Vol. 347.

[5] A. J. den Boef, Optical wafer metrology sensors for process-robust $\mathrm{CD}$ and overlay control in semiconductor device manufacturing, Surf. Topography: Metrol. Properties 4, 023001 (2016).

[6] C. Thomsen, H. T. Grahn, H. J. Maris, and J. Tauc, Surface generation and detection of phonons by picosecond light pulses, Phys. Rev. B 34, 4129 (1986).

[7] O. Matsuda, M. C. Larciprete, R. L. Voti, and O. B. Wright, Fundamentals of picosecond laser ultrasonics, Ultrasonics 56, 3 (2015).

[8] P. Ruello and V. E. Gusev, Physical mechanisms of coherent acoustic phonons generation by ultrafast laser action, Ultrasonics 56, 21 (2015).

[9] T. F. Crimmins, A. A. Maznev, and K. A. Nelson, Transient grating measurements of picosecond acoustic pulses in metal films, Appl. Phys. Lett. 74, 1344 (1999).

[10] A. Devos, Colored ultrafast acoustics: From fundamentals to applications, Ultrasonics 56, 90 (2015). 
[11] O. B. Wright and K. Kawashima, Coherent Phonon Detection from Ultrafast Surface Vibrations, Phys. Rev. Lett. 69, 1668 (1992).

[12] M. Kouyaté, T. Pezeril, V. Gusev, and O. Matsuda, Theory for optical detection of picosecond shear acoustic gratings, J. Opt. Soc. Am. B 33, 2634 (2016).

[13] R. M. Slayton and K. A. Nelson, Picosecond acoustic transmission measurements. I. Transient grating generation and detection of acoustic responses in thin metal films, J. Chem. Phys. 120, 3908 (2004).

[14] T. Saito, O. Matsuda, and O. B. Wright, Picosecond acoustic phonon pulse generation in nickel and chromium, Phys. Rev. B 67, 205421 (2003).

[15] O. B. Wright and V. E. Gusev, Acoustic generation in crystalline silicon with femtosecond optical pulses, Appl. Phys. Lett. 66, 1190 (1995).

[16] T. Saito, O. Matsuda, M. Tomoda, and O. B. Wright, Imaging gigahertz surface acoustic waves through the photoelastic effect, J. Opt. Soc. Am. B 27, 2632 (2010).

[17] R. M. Slayton, K. A. Nelson, and A. A. Maznev, Transient grating measurements of film thickness in multilayer metal films, J. Appl. Phys. 90, 4392 (2001).

[18] M. Lejman, V. Shalagatskyi, O. Kovalenko, T. Pezeril, V. V. Temnov, and P. Ruello, Ultrafast optical detection of coherent acoustic phonons emission driven by superdiffusive hot electrons, J. Opt. Soc. Am. B 31, 282 (2014).

[19] K. E. O'Hara, X. Hu, and D. G. Cahill, Characterization of nanostructured metal films by picosecond acoustics and interferometry, J. Appl. Phys. 90, 4852 (2001).

[20] D. Yarotski, E. Fu, L. Yan, Q. Jia, Y. Wang, A. J. Taylor, and B. P. Uberuaga, Characterization of irradiation damage distribution near $\mathrm{TiO}_{2} / \mathrm{SrTiO}_{3}$ interfaces using coherent acoustic phonon interferometry, Appl. Phys. Lett. 100, 251603 (2012).

[21] A. Steigerwald, Y. Xu, J. Qi, J. Gregory, X. Liu, J. K. Furdyna, K. Varga, A. B. Hmelo, G. Lüpke, L. C. Feldman, and N. Tolk, Semiconductor point defect concentration profiles measured using coherent acoustic phonon waves, Appl. Phys. Lett. 94, 111910 (2009).

[22] T. Dehoux, K. Ishikawa, P. H. Otsuka, M. Tomoda, O. Matsuda, M. Fujiwara, S. Takeuchi, I. A. Veres, V. E. Gusev, and O. B. Wright, Optical tracking of picosecond coherent phonon pulse focusing inside a sub-micron object, Light: Sci. Appl. 5, e16082 (2016).

[23] K. A. Nelson, R. J. D. Miller, D. R. Lutz, and M. D. Fayer, Optical generation of tunable ultrasonic waves, J. Appl. Phys. 53, 1144 (1982).

[24] T. Pezeril, P. Ruello, S. Gougeon, N. Chigarev, D. Mounier, J.-M. Breteau, P. Picart, and V. Gusev, Generation and detection of plane coherent shear picosecond acoustic pulses by lasers: Experiment and theory, Phys. Rev. B 75, 174307 (2007).

[25] P. Babilotte, P. Ruello, D. Mounier, T. Pezeril, G. Vaudel, M. Edely, J.-M. Breteau, V. Gusev, and K. Blary, Femtosecond laser generation and detection of high-frequency acoustic phonons in GaAs semiconductors, Phys. Rev. B 81, 245207 (2010).

[26] O. B. Wright, Ultrafast nonequilibrium stress generation in gold and silver, Phys. Rev. B 49, 9985 (1994).

[27] B. Bonello, B. Perrin, E. Romatet, and J. Jeannet, Application of the picosecond ultrasonic technique to the study of elastic and time-resolved thermal properties of materials, Ultrasonics 35, 223 (1997).

[28] F. Pérez-Cota, R. J. Smith, E. Moradi, L. Marques, K. F. Webb, and M. Clark, High resolution 3D imaging of living cells with sub-optical wavelength phonons, Sci. Rep. 6, 1 (2016).

[29] J. A. Guggenheim, J. Li, T. J. Allen, R. J. Colchester, S. Noimark, O. Ogunlade, I. P. Parkin, I. Papakonstantinou, A. E. Desjardins, E. Z. Zhang, and P. C. Beard, Ultrasensitive plano-concave optical microresonators for ultrasound sensing, Nat. Photonics 11, 714 (2017).

[30] J. A. Guggenheim, T. J. Allen, A. Plumb, E. Z. Zhang, M. Rodriguez-Justo, S. Punwani, and P. C. Beard, Photoacoustic imaging of human lymph nodes with endogenous lipid and hemoglobin contrast, J. Biomed. Opt. 20, 1 (2015).

[31] J. Brunker and P. Beard, Velocity measurements in whole blood using acoustic resolution photoacoustic doppler, Biomed. Opt. Express 7, 2789 (2016).

[32] A. P. Jathoul, J. Laufer, O. Ogunlade, B. Treeby, B. Cox, E. Zhang, P. Johnson, A. R. Pizzey, B. Philip, T. Marafioti, M. F. Lythgoe, R. B. Pedley, M. A. Pule, and P. Beard, Deep in vivo photoacoustic imaging of mammalian tissues using a tyrosinase-based genetic reporter, Nat. Photonics 9, 239 (2015).

[33] G. Leahu, E. Petronijevic, A. Belardini, M. Centini, R. Li Voti, T. Hakkarainen, E. Koivusalo, M. Guina, and C. Sibilia, Photo-acoustic spectroscopy revealing resonant absorption of self-assembled GaAs-based nanowires, Sci. Rep. 7, 2833 (2017).

[34] E. Petronijevic, G. Leahu, V. Mussi, C. Sibilia, and F. A. Bovino, Photoacoustic technique for the characterization of plasmonic properties of $2 \mathrm{~d}$ periodic arrays of gold nanoholes, AIP Adv. 7, 025210 (2017).

[35] T. Pozar, J. Lalos, A. Babnik, R. Petkovsek, M. BethuneWaddell, K. J. Chau, G. V. B. Lukasievicz, and N. G. C. Astrath, Isolated detection of elastic waves driven by the momentum of light, Nat. Commun. 9, 3340 (2018).

[36] P. Burgholzer, M. Thor, J. Gruber, and G. Mayr, Threedimensional thermographic imaging using a virtual wave concept, J. Appl. Phys. 121, 105102 (2017).

[37] T. W. Murray, M. Haltmeier, T. Berer, E. LeissHolzinger, and P. Burgholzer, Super-resolution photoacoustic microscopy using blind structured illumination, Optica 4, 17 (2017).

[38] N. Mokhlesi and R. Scheuerlein, Three dimensional nand memory, U.S. Patent No. US7851851B2 (2007).

[39] H. Zhang, A. Antoncecchi, S. Edward, I. Setija, P. Planken, and S. Witte, Unraveling Phononic, Optoacoustic, and Mechanical Properties of Metals with Light-Driven Hypersound, Phys. Rev. Appl. 13, 014010 (2020).

[40] S. Edward, A. Antoncecchi, H. Zhang, H. Sielcken, S. Witte, and P. C. M. Planken, Detection of periodic structures through opaque metal layers by optical measurements of ultrafast electron dynamics, Opt. Express 26, 23380 (2018).

[41] J. Hohlfeld, S.-S. Wellershoff, J. Güdde, U. Conrad, V. Jähnke, and E. Matthias, Electron and lattice dynamics following optical excitation of metals, Chem. Phys. 251, 237 (2000). 
[42] R. H. M. Groeneveld, R. Sprik, and A. Lagendijk, Femtosecond spectroscopy of electron-electron and electronphonon energy relaxation in Ag and Au, Phys. Rev. B 51, 11433 (1995).

[43] H. E. Elsayed-Ali, T. Juhasz, G. O. Smith, and W. E. Bron, Femtosecond thermoreflectivity and thermotransmissivity of polycrystalline and single-crystalline gold films, Phys. Rev. B 43, 4488 (1991).

[44] H. N. Lin, R. J. Stoner, H. J. Maris, and J. Tauc, Phonon attenuation and velocity measurements in transparent materials by picosecond acoustic interferometry, J. Appl. Phys. 69, 3816 (1991).

[45] A. Devos, R. Côte, G. Caruyer, and A. Lefèvre, A different way of performing picosecond ultrasonic measurements in thin transparent films based on laser-wavelength effects, Appl. Phys. Lett. 86, 211903 (2005).

[46] O. Matsuda, T. Pezeril, I. Chaban, K. Fujita, and V. Gusev, Time-domain Brillouin scattering assisted by diffraction gratings, Phys. Rev. B 97, 064301 (2018).

[47] V. E. Gusev and P. Ruello, Advances in applications of time-domain Brillouin scattering for nanoscale imaging, Appl. Phys. Rev. 5, 031101 (2018).

[48] S. M. Nikitin, N. Chigarev, V. Tournat, A. Bulou, D. Gasteau, B. Castagnede, A. Zerr, and V. E. Gusev, Revealing sub-mm and mm-scale textures in $\mathrm{H}_{2} \mathrm{O}$ ice at megabar pressures by time-domain Brillouin scattering, Sci. Rep. 5, 9352 (2015).
[49] A. Stovas and B. Ursin, Equivalent time-average and effective medium for periodic layers, Geophys. Prospect. 55, 871 (2007).

[50] S. Anisimov, B. Kapeliovich, and T. Perel-man, Electron emission from metal surfaces exposed to ultrashort laser pulses, J. Exp. Theor. Phys. 66, 375 (1974).

[51] J. Güdde, J. Hohlfeld, J. Müller, and E. Matthias, Damage threshold dependence on electron-phonon coupling in $\mathrm{Au}$ and Ni films, Appl. Surf. Sci. 127-129, 40 (1998).

[52] M. Bonn, D. N. Denzler, S. Funk, M. Wolf, S.-S. Wellershoff, and J. Hohlfeld, Ultrafast electron dynamics at metal surfaces: Competition between electron-phonon coupling and hot-electron transport, Phys. Rev. B 61, 1101 (2000).

[53] S.-S. Wellershoff, J. Güdde, J. Hohlfeld, J. Muller, and E. Matthias, Role of electron-phonon coupling in femtosecond laser damage of metals, Proc. SPIE 3343, 378 (1998).

[54] C. Suárez, W. E. Bron, and T. Juhasz, Dynamics and Transport of Electronic Carriers in Thin Gold Films, Phys. Rev. Lett. 75, 4536 (1995).

[55] J. L. Hostetler, A. N. Smith, D. M. Czajkowsky, and P. M. Norris, Measurement of the electron-phonon coupling factor dependence on film thickness and grain size in $\mathrm{Au}, \mathrm{Cr}$, and Al, Appl. Opt. 38, 3614 (1999).

[56] P. B. Johnson and R. W. Christy, Optical constants of transition metals: Ti, V, Cr, Mn, Fe, Co, Ni, and Pd, Phys. Rev. B 9, 5056 (1974). 